\title{
Data Collapse of Energy Loss in Soft Magnetic Materials as a Way for Testing Measurement Set
}

\author{
K. SOKAlSkI* AND J. SZCZYGŁOWSKI \\ Faculty of Electrical Engineering, Technical University of Częstochowa \\ al. Armii Krajowej 17, 42-200 Czȩstochowa, Poland
}

(Received October 26, 2009; revised version December 6, 2009; in final form December 15, 2009)

\begin{abstract}
Basing on the data collapse of the energy loss in soft magnetic materials, we propose a dimensionless measure of measurement set's uncertainty. The derived measure enables to compare uncertainty of different measurement sets and comparison of measurement data.
\end{abstract}

PACS numbers: 75.50.-y, 89.75.Da

\section{Introduction}

In 1995 the leading European Laboratories busy with measurements of the electrical steel magnetic properties were trying to compare the measurement results of the power loss in electrical sheet steel under the conditions of rotating and alternating flux [1]. The six participating laboratories applied different measurement methods, similar only in some cases. According to this report the results for non-oriented materials were in fairly good agreement, whereas for grain-oriented materials the results differed considerably. The observed dispersion was attributed to several factors. For the purposes of this paper we distinguish the two of them: the geometry of the sample (size, shape) and the way for assessment of uncertainty contributions. Taking from [1] the idea of the inter-comparison of measurement data of the energy losses in soft magnetic materials (SMM), we perform such an inter-comparison with data taken in two laboratories [2-4], however under the conditions of axial and alternating flux. We show that the interference of the sample's geometry can be scaled off and this factor does not interfere on the data inter-comparison. Moreover, the material type can be scaled off from the dependence of energy losses vs. the peak of induction and magnetizing frequencies. This property of SMM allows comparing different measurement sets. Successively, this fact allows introducing an absolute measure of uncertainty characterizing the given measurement set. Therefore, the way for assessing the uncertainty contributions would not interfere with the above mentioned data comparison.

\footnotetext{
* corresponding author; e-mail: sokalski@el.pcz.czest.pl
}

\section{Data collapse}

In 2006 the scaling theory applied to phenomenological description of energy loss in soft magnetic materials led to the following formula for $P_{\text {tot }}$ [2]:

$$
P_{\mathrm{tot}}=B_{\mathrm{m}}^{\beta}\left[\Gamma^{(1)} \frac{f}{B_{\mathrm{m}}^{\alpha}}+\Gamma^{(2)}\left(\frac{f}{B_{\mathrm{m}}^{\alpha}}\right)^{2}+\ldots\right],
$$

where $f$ - frequency, $B_{\mathrm{m}}$ - amplitude of magnetic field induction. Values of $\alpha, \beta$ and amplitudes $\Gamma^{(n)}$ for ten selected soft magnetic materials have been estimated. For all investigated samples, only two first terms of (1) were significant, predicting monotonic increase of $P_{\text {tot }}$ vs. $f$. Recently Eq. (1) has been confirmed empirically by Yuan et al. [3]. In the same period the further confirmation of the scaling in SMM, as well as its mathematical and physical background, have been presented by the authors of this paper [4]. Due to the second degree of (1), it was possible to obtain the data collapse by the appropriate scaling. Accordingly, Eq. (1) was transformed to the sample-independent form, which includes the scaled variables $\widetilde{P}_{\text {tot }}$ and $\widetilde{f}$ :

$$
\widetilde{P}_{\text {tot }}=\tilde{f}+\widetilde{f}^{2}
$$

where

$$
\widetilde{P}_{\text {tot }}=\frac{\Gamma^{(2)}}{\Gamma^{(1)^{2}}} \frac{P_{\text {tot }}}{B_{\mathrm{m}}^{\beta}}, \quad \tilde{f}=\frac{\Gamma^{(2)}}{\Gamma^{(1)}} \frac{f}{B_{\mathrm{m}}^{\alpha}} .
$$

The chart of all measurement data, scaled according to (3), confirms the data collapse for total energy loss in soft magnetic materials. Figure 1 depicts the revealed data collapse on the basis of nine samples.

\subsection{Data's scaling and inter-comparison}

For inter-comparison we have selected two sets of energy losses data. The first one belongs to Yuan 


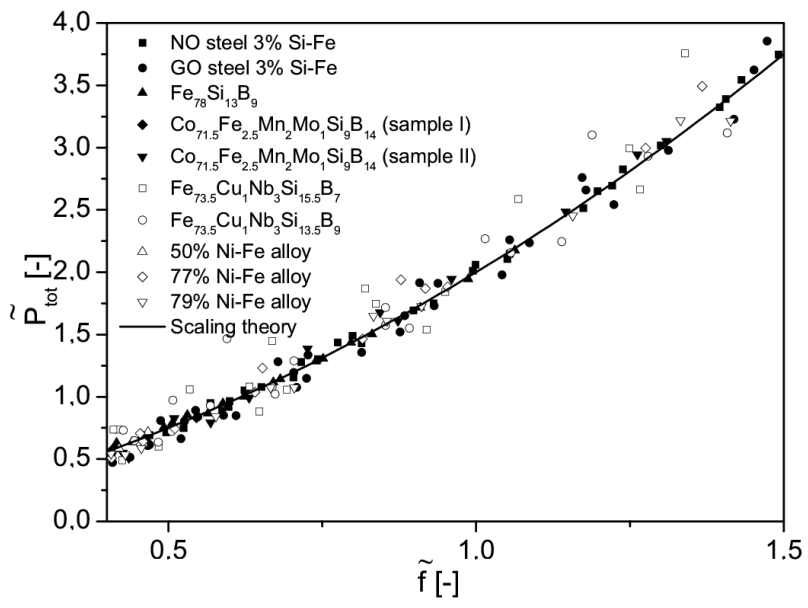

Fig. 1. Part of a distribution of measurements around theoretical curve according to Eqs. (2), (3) [2].

et al. [3] and consists of the three sets of data's measurements of Fe-based amorphous alloys: $S_{1}=$ $\mathrm{Fe}_{76} \mathrm{Mo}_{2} \mathrm{Si}_{2} \mathrm{P}_{10} \mathrm{C}_{7.5} \mathrm{~B}_{2.5}, S_{2}=\mathrm{Fe}_{79.8} \mathrm{Mo}_{2.1} \mathrm{Si}_{2.1} \mathrm{P}_{8} \mathrm{C}_{6} \mathrm{~B}_{2}$ and $S_{3}=\mathrm{Fe}_{80} \mathrm{Mo}_{1} \mathrm{Si}_{2} \mathrm{P}_{8} \mathrm{C}_{6} \mathrm{~B}_{3}$.

The samples were thin ribbons wound into toroids. For details concerning measurement method of the energy loss in these samples we refer the reader to Ref. [3]. On the basis of measured data the parameters' values of (1) have been estimated (see Table I, after [3]).

TABLE I

Scaling exponents and coefficients of (1).

\begin{tabular}{c|c|c|c|c}
\hline \hline Sample & $\alpha$ & $\beta$ & $\Gamma^{(1)}\left[\frac{\mathrm{m}^{2}}{\mathrm{~s}^{2}} T^{\alpha-\beta}\right]$ & $\Gamma^{(2)}\left[\frac{\mathrm{m}^{2}}{\mathrm{~s}} T^{\alpha-\beta}\right]$ \\
\hline$S_{1}^{*}$ & -1.533 & -0.319 & $6.744 \times 10^{-3}$ & $1.322 \times 10^{-6}$ \\
$S_{2}$ & -0.364 & 1.259 & $1.412 \times 10^{-2}$ & $1.917 \times 10^{-6}$ \\
$S_{3}$ & -0.504 & 1.069 & $9.11 \times 10^{-3}$ & $3.389 \times 10^{-6}$ \\
\hline$P_{1}$ & -2.9448 & -1.7761 & $2.90 \times 10^{-3}$ & $4.60 \times 10^{-6}$ \\
$P_{2}$ & -1.5190 & -0.3754 & $2.53 \times 10^{-3}$ & $6.79 \times 10^{-6}$ \\
$P_{3}$ & -3.2309 & -1.3649 & $3.22 \times 10^{-4}$ & $1.95 \times 10^{-7}$ \\
\hline
\end{tabular}

* The data for $S_{1}, S_{2}$, and $S_{3}$ have been kindly supplied by the authors of [3].

The second set contains some of our results $[2,4]$ for the energy losses in the following alloys: amorphous ribbon $P_{1}=\mathrm{Fe}_{78} \mathrm{Si}_{13} \mathrm{~B}_{9}$, Co-based amorphous alloy $P_{2}=\mathrm{Co}_{71.5} \mathrm{Fe}_{2.5} \mathrm{Mn}_{2} \mathrm{Mo}_{1} \mathrm{Si}_{9} \mathrm{~B}_{14}$, nanocrystalline alloy $P_{3}=\mathrm{Fe}_{73.5} \mathrm{Cu}_{1} \mathrm{Nb}_{3} \mathrm{Si}_{15.5} \mathrm{~B}_{7}$.

The corresponding scaling exponents $\alpha, \beta$ and the scaling coefficients $\Gamma^{(1)}, \Gamma^{(2)}$ are presented in Table I.

\subsection{Assessment of uncertainty}

Plotting $\widetilde{P}_{\text {tot }}$ versus $\tilde{f}$ for the all considered samples (Fig. 2), we confirm that the data collapse takes place for the selected samples. Since all the magnitudes in

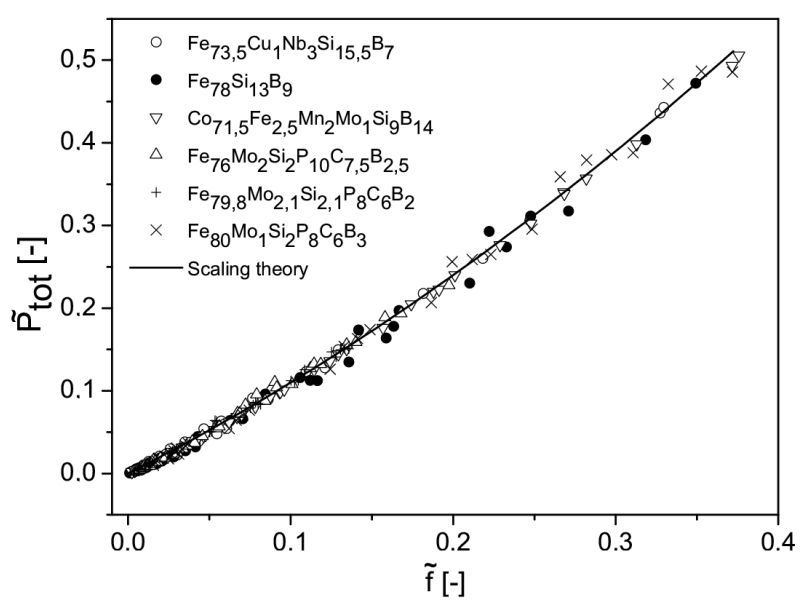

Fig. 2. The data collapse for total energy losses of compared materials.

TABLE II

Comparisons of uncertainty measures.

\begin{tabular}{c|c|c|c|c|c}
\hline \hline Sample & $N_{i}$ & $\widetilde{f}_{\max }[-]$ & $D_{i}^{2}[-]$ & $D_{\text {tot }}^{2}[-]$ & $\sqrt{\bar{D}_{\text {tot }}^{2}}[-]$ \\
\hline$S_{1}$ & 48 & 0.197 & $1.64 \times 10^{-5}$ & & \\
$S_{2}$ & 40 & 0.136 & $7.42 \times 10^{-5}$ & $6.57 \times 10^{-5}$ & $8.11 \times 10^{-3}$ \\
$S_{3}$ & 32 & 0.371 & $1.29 \times 10^{-4}$ & & \\
\hline$P_{1}$ & 48 & 0.349 & $9.23 \times 10^{-5}$ & & \\
$P_{2}$ & 47 & 0.376 & $2.05 \times 10^{-5}$ & $4.26 \times 10^{-5}$ & $6.53 \times 10^{-3}$ \\
$P_{3}$ & 49 & 0.329 & $1.53 \times 10^{-5}$ & &
\end{tabular}

(2) are dimensionless and the formula for $\widetilde{P}_{\text {tot }}$ is sample-independent, we propose to introduce a measure of uncertainty characterizing the measurement set by the total distance of all empirical points from the scaling curve (2):

$$
D=\sqrt{\frac{1}{N} \sum_{i}^{N}\left[\widetilde{P}_{\mathrm{tot}}^{\mathrm{em}}\left(\widetilde{f}_{i}\right)-\widetilde{P}_{\mathrm{tot}}^{\mathrm{th}}\left(\widetilde{f}_{i}\right)\right]^{2}},
$$

where $\widetilde{P}_{\text {tot }}^{\text {em }}$ and $\widetilde{P}_{\text {tot }}^{\text {th }}$ are empirically and theoretically scaled losses, respectively. $N$ is the number of exprimental points. Comparisons of uncertainty measures are presented in Table II. $D_{\text {tot }}^{2}$ is the average of $D_{i}^{2}$ calculated for three samples belonging to either of the two selected sets

$$
D_{\text {tot }}^{2}=\sum_{i=1}^{3} \frac{N_{i}}{N_{1}+N_{2}+N_{3}} D_{i}^{2} .
$$

Comparing $\sqrt{D_{\text {tot }}^{2}}$ for the both data sets one can see that the values of uncertainties characterizing the compared measurement sets are very close. Processing data for the presented comparison, we satisfied the following conditions:

- The higher order terms for $n>2$ in (1) are neglected. Applying to $P_{\text {tot }}$ the scaling hypothesis $[2,4]$, we have derived the scaled formula (1) in 
the form of infinite series. In most cases this series converges very fast. Moreover, in many cases Eq. (1) can be truncated at the second order term which means that two first terms are sufficient. Basis for the described concept here is the data collapse, therefore it is important to mention that the collapse occurs only for data obeying the second degree formula (1), whereas the higher terms limit applicability of this paper to a range of small values of $\widetilde{f}$.

- The scaled data $\left(\widetilde{f}, \widetilde{P}_{\text {tot }}\right)$ for the measuremet sets to be compared, should be of the comparable lengths: $N_{i} \approx N_{j}$.

- The ranges of scaled frequency $\widetilde{f}$ should be comparable as well. The frequency range, in all described cases is an interval: $\left[0, \widetilde{f}_{\max }\right]$, therefore the values of $\tilde{f}_{\max }$ have to be comparable. We can see from Figs. 1 and 2 that the fluctuations of the scaled measurement data around the universal curve (2) increase with increasing $\widetilde{f}$. For this reason the distributions of density of the measured points around (2) should be comparable functions of $\tilde{f}$.

- Estimates of the scaling parameters $\alpha, \beta, \Gamma^{(1)}$ and $\Gamma^{(2)}$ should be as accurate as possible. The precision of their values is important, since $D_{i}^{2}$, being an uncertainty measure, sensitively depends on them.

\section{Conclusions}

Progress in the design of electrical machines and transformers depends on the comprehensive knowledge of magnetic material properties under standard and non-standard excitation conditions. However, an agreed standardized method does not exist and the reproducibilities of the different methods used in different laboratories are unknown. We are of the opinion that the reason of such situation is lacking of statistical method enabling the appropriate data's intercomparison. In this paper we have proposed a solution of this problem. As we have shown, the data collapse supplies such a method and enables to introduce an universal measure of uncertainty which compares different experimental sets even basing on different methods. Therefore, the introduced method also can serve as a tool to compare measurement data obtained in different laboratories. The measure (5) expresses the total uncertainty characterizing the measurement set in the chosen range of $\tilde{f}$. There are three contributions to $D_{\text {tot }}$ resulting from: (1) uncertainty characterizing the measurement method and construction of the measurement set, (2) uncertainty of measurements of elementary magnitudes, (3) errors resulting from the approximation (1) and estimations of $\alpha, \beta, \Gamma^{(1)}$ and $\Gamma^{(2)}$.

The method can be applied to investigations of any phenomenon satisfying the scaling hypothesis and the corresponding measurement data, satisfying the conditions given in Sect. 2.2.

\section{Acknowledgments}

The authors would like to thank W.J. Yuan, F.J. Liu, S.J. Pang, Y.J. Song, and T. Zhang for sharing their data. This paper has been done according to the project N N510193138, being considered by the Polish Ministry of Science and Higher Education.

\section{References}

[1] J. Sievert, H. Ahlers, M. Birkfeld, B. Cornut, F. Fiorillo, K.A. Hempel, T. Kochmann, A. Kedous-Lebouc, T. Meydan, A. Moses, A.M. Rietto, J. Magn. Magn. Mater. 160, 115 (1996).

[2] K. Sokalski, J. Szczygłowski, M. Najgebauer, W. Wilczyński, COMPEL: Int. J. Comput. Math. Electr. Electron. Eng. 26, 640 (2007).

[3] W.J. Yuan, F.J. Liu, S.J. Pang, Y.J. Song, T. Zhang, Intermetallics 17, 278 (2009).

[4] K. Sokalski, J. Szczygłowski, Acta Phys. Pol. A 115, 920 (2009). 